\title{
Study on Preparation of Ultrahigh Molecular Weight Polyethylene Fibers
}

\author{
Wei $\mathrm{Li}^{1}$, Haiyan $\mathrm{Hu}^{2}$ \\ ${ }^{1}$ Science School, Tibet University, Lhasa, 850000 \\ ${ }^{2}$ Kai Hui Pharmaceutical (Shanghai) Co., Ltd. Shanghai, 201499
}

Keywords: Ultrahigh Molecular, Weight Polyethylene Fibers, Preparation

\begin{abstract}
Ultra High Molecular Weight Polyethylene (UHMWPE) fibers are widely used in all walks of life because of their excellent properties and have attracted the attention of many experts and scholars at home and abroad. Research and preparation of domestic UHMWPE fiber has been for decades, although great progress has been made, but overall, the domestic UHMWPE fiber production is small, low quality and stability needs to be further improved. Therefore, it is very important to study the preparation of UHMWPE fiber and the mechanism of its microstructure evolution.
\end{abstract}

\section{Introduction}

Ultra High Molecular Weight Polyethylene (UHMWPE) is widely used in many military and civilian applications due to its range of excellent properties such as composites, ropes, textiles, joints, racquets, body armor, belts Wait. UHMWPE fibers have high specific strength and modulus, excellent chemical resistance, low moisture absorption, good impact and abrasion resistance, weather resistance and lower density than water.

The reason why UHMWPE fiber has excellent performance, which is closely related to its molecular structure. (1) UHMWPE fiber molecular weight is usually between 100 to 6 million, the molecular linear high degree of orientation of the straight chain structure; (2) Polyethylene fiber from vinyl polymerization of linear long-chain polymer, polyethylene molecular chain Space arranged in a flat zigzag shape, the key angle of $109.3^{\circ}$; (3) molecular symmetry of non-polar groups and no side chain, so the intermolecular force is small; (4) polyethylene molecular chain of good flexibility and regularity of molecules The chain can be repeatedly folded and neatly stacked to form crystals. The high-tension and high-tension high-modulus UHMWPE fibers are obtained through the high-speed drawing to make the preferred orientation of the macromolecules.

\section{Ultra-High Molecular Weight Polyethylene Applications}

UHMWPE fibers have higher relative molecular mass, high crystallinity and high orientation, and have straight chain structure. This special structure gives excellent physical and mechanical properties of UHMWPE fiber, which makes UHMWPE fiber have high strength and high modulus, excellent mechanical properties, Bending ability, abrasion resistance, resistance to acid and alkali corrosion of chemical substances, non-conductive, high impact strength and other excellent performance. In industrial, military, medical, sports, aviation, Hainan Airlines and many other areas play an important role. In the industry, UHMWPE fiber composite materials can be used as conveyor belts, plastic buckets, buffer equipment and so on. In sports, UHMWPE fiber can be used in bicycles, all kinds of racquets, ski boards, struts, diving boards, skis and so on. Medically, UHMWPE composites can be used for a wide range of joint applications and a great deal of research has been conducted. Doudin $\mathrm{K}$ with a stable antioxidant vitamin $\mathrm{E}$ cross-linking reaction to improve the UHMWPE composite wear resistance of artificial joints. Militarily, UHMWPE composites can be used on body armor, bullet-proof armor, fighter aircraft and the like and can reduce the weight of weapons. In the study of body armor, Peng $\mathrm{Y}$ et al. Explored burning armor-piercing projectiles to shoot $40-130 \mathrm{~mm}$ UHP-BASFRC rear cloth (UHMWPE composite) at a speed of $810 \mathrm{~m} / \mathrm{s}$. The body armor remains intact. UHMWPE fibers are also used in the 
treatment of heavy metal pollution. Qinghong Guo et al. In a simulated seawater, a series of chemical modifications are used to selectively adsorb UHMWPE. Amorphous oxime (AO) is attached to the UHMWPE fibers for selective adsorption Simulate seawater in the uranium, vanadium, nickel, lead and other heavy metals. Among them, uranium is most adsorbed, which has important practical significance for the treatment of heavy metal pollution in seawater.

\section{Ultra-High Molecular Weight Polyethylene Fiber Preparation}

Ultra-high molecular weight polyethylene fibers, the molecular chain is highly oriented crystalline straight chain structure, this special structure gives UHMWPE fiber excellent physical and mechanical properties. UHMWPE fiber is widely used in all walks of life, but also is an indispensable strategic material in national security and economic construction, so many experts and scholars at home and abroad are concerned about the research. Domestic research UHMWPE fiber preparation for decades, although made great progress, but overall, domestic UHMWPE fiber production, low quality and stability also need to be improved. Therefore, it is of great significance to study the preparation process of UHMWPE fiber to improve the fiber properties. At present, the mature preparation method is the preparation of UHMWPE fiber by the jelly spinning method. The main steps of jelly spinning are divided into dissolution of UHMWPE, twin-screw extrusion, spinning, extraction drying, multi-stage drafting, and finally to obtain the finished fiber, wherein the dissolution of raw materials is the most crucial factor for preparing high-performance fiber. Due to its high molecular weight, high viscosity and high number of entangled points, UHMWPE makes it difficult for UHMWPE powders to dissolve rapidly and effectively in white oil solvents. Therefore, the production efficiency of UHMWPE fibers is low. The traditional production process requires high temperature $\left(290^{\circ} \mathrm{C}\right)$ spinning, high temperature spinning UHMWEP molecular weight greatly degraded, thus affecting the fiber properties. In this thesis, the modified UHMWPE raw materials are prepared by using the technological route with independent intellectual property rights. The modified raw materials have the characteristics of low crystallinity, low entanglement and the like, and can be rapidly and effectively dissolved in white oil solvent at low temperature and spun in low temperature Silk effectively alleviate the degradation of UHMWPE, laying the foundation for the preparation of high performance UHMWPE fiber. In the Donghua University small-scale production line, the use of modified UHMWPE raw materials, with jelly spinning preparation of ultra-high molecular weight polyethylene fibers, high-performance fiber UHMWPE fiber technology research. And then use its process parameters, in the large-scale exploration of industrial production of high-performance ultra-high molecular weight polyethylene fibers. The mechanical properties of ultra-high molecular weight polyethylene fibers during the preparation of UHMWPE fibers by the jelly spinning method are as follows: raw material dissolution, solvent selection, spinning solution concentration, spinning temperature, twin-screw extrusion speed, coagulation bath and coagulation The choice of bath temperature, the choice of extractant, the factors of drafting. The factors of drafting include the speed of stretching, stretching temperature and stretching ratio.

\section{Factors Affecting Jelly Spinning UHMWPE Fiber}

The dissolution of raw materials is the most critical factor in the preparation of high-performance UHMWPE fibers. UHMWPE molecular weight is generally higher than 4 million, so conventional UHMWPE molecules easily entangled together lead to the viscosity of the liquidity is poor, more impact on the UHMWPE entanglement points after the drafting multiple and drafting effect. Therefore, conventional UHMWPE powder is not suitable as a raw material for UHMWPE fibers. In order to solve the problem of entanglement in the preparation of UHMWPE, UHMWPE fiber can be quickly and effectively dissolved. In this thesis, a modified UHMWPE raw material was prepared by using patented technology with intellectual property. The modified UHMWPE could be rapidly entangled and dissolved rapidly in the solvent at low temperature, effectively alleviating the degradation of UHMWPE, and thus prepared high performance UHMWPE fiber. In this paper, XRD, DSC and SEM tests of UHMWPE raw materials before and after modification were carried 
out, and the dissolution experiments of modified UHMWPE powder were explored.

A variety of solvents are used to dissolve UHMWPE powder to produce high strength UHMWPE fibers, including: dodecane, decalin, p-xylene, 1,2,4-trichlorobenzene, kerosene and paraffin oil. Xudong Fang and other polybutene (PB) as a solvent, formulated as 2wt\% UHMWPE dilute solution to prepare a strength of 4Gpa, modulus of 150GPa UHMWPE fiber. Among various solvents, decahydronaphthalene has the best solubility and volatility. The frozen collagen fiber obtained by extraction can be directly stretched without the extraction process, but it is expensive and the industrial production cost is too high. Considering the cost of industrialization and the current experimental conditions, this paper uses paraffin oil from Hangzhou refinery as the solvent for preparing UHMWPE fiber.

The ratio of the ultra-high molecular weight polyethylene raw materials and the solvent concentration has an important impact on the preparation of UHMWPE fiber. The concentration of spinning solution determines the number of entanglement points between polyethylene macromolecules, while the molecular entanglement points of polyethylene directly affect the fiber drawing effect, thereby affecting the fiber properties. If the spinning solution concentration is too high, too many macromolecular entanglement points, the tensile resistance increases, the fiber can not achieve high draw ratio; spinning solution concentration is too high, polyethylene can not be successfully dissolved completely affect the performance of frozen jelly; If the spinning solution concentration is too low, the entanglement of macromolecules is too small, it will form a pile of lamellar crystal structure, macromolecular chains slip during stretching. Therefore, the concentration of spinning solution to be appropriate, the appropriate entanglement of macromolecular chains, drafting can successfully transfer stress, stretching multiple and stretching effect will be more excellent. In this paper, modified UHMWPE as raw material to explore the concentration of spinning solution were $6 \%, 7 \%, 8 \%, 9 \%, 10 \%$ of the preparation of high-performance UHMWPE fiber.

The twin-screw extrusion rate determines the size of the twin-screw shear force, the twin-screw rate is too small, the shear force on the polyethylene molecule is too small, and the polyethylene molecules can not be thoroughly mixed and dissolved in the white oil solvent. In addition, the residence time of the polyethylene molecule is long, the molecular weight degradation exacerbated; at the same time the shear force is too small, can not fully make the tangle of the molecular chain untied, the fiber in the subsequent drawing process is easy to break; if the twin-screw extrusion rate is too high, will destroy the molecular structure of polyethylene polyethylene molecules degradation, entanglement is too small and thus affect the production efficiency. Suitable twin-screw extrusion rate to produce high-performance UHMWPE fiber. This project explored the effect of twin-screw extrusion rate on the mechanical properties of UHMWPE fiber at 4r / $\mathrm{min}, 6 \mathrm{r} / \mathrm{min}, 8 \mathrm{r} / \mathrm{min}, 10 \mathrm{r} /$ min and $12 \mathrm{r} / \mathrm{min}$, respectively.

There is a large amount of solvent in the UHMWPE jelly fibroin prepared by jelly spinning method, which makes the tensile properties of the fiber worse. It can not be directly stretched by ultrapure and must be extracted before stretching. The UHMWPE jelly fiber, which is formed by jelly spinning, contains a large amount of solvent, and the network structure is extremely loose. The interaction between the polyethylene macromolecules in the network has become very small due to the dissolution of the solvent. It is therefore difficult to form a stable stretching ratio, affecting the strength and modulus, so extraction is performed before drawing to remove a large amount of diluent.

In the jelly spinning process, UHMWPE spinning solution through the spinneret hole after quenching in the coagulation bath to quench the temperature below the gel, making it a jelly thread. In the frozen collagen filaments, almost all of the solvent is contained therein, so that the unwound state of the UHMWPE macromolecule chains is well maintained and the solution temperature drops resulting in the formation of folded chains of UHMWPE in the gel. In other words, the formation of uncoiled state of the folded chain pieces, which laid the foundation for the ultra-tensile stretching of the original collagen gel. In general, water can be very good heat exchange with the frozen collagen fibers, so that it quenches, so the experiment selected water as the coagulation bath. If the cooling 
temperature is too high, the jelly fiber crystallinity, lamellar thickness and growth cycle becomes larger, so that the jelly point in the jelly collagen fibers less, resulting in poor post-stretch effect. After research shows that the cooling temperature is generally below $50{ }^{\circ} \mathrm{C}$. In this experiment, cold gel strands are cooled with water at room temperature to save cost.

For high strength, high modulus UHMWPE fibers, in addition to the unwinding of the UHMWPE macromolecular chains in the spinning solution, another key to high strength, high modulus UHMWPE fibers lies in the ultra-extensional stretching of jelly fibers. The elongation of fiber has important influence on the mechanical properties, crystallinity and thermal properties of the fiber. In the process of high-power stretching, the semi-crystalline microfibrils of the frozen collagen filaments are transformed into highly oriented and highly crystalline structures of the stretched chains to produce fibers of high strength and high modulus. The extracted dry jelly fibers, due to solvation, are far from being eliminated. Therefore, the bulk density of the macromolecule folding chain-like crystals is very low and remains a loose large network structure, and the fibers are easily elongated. As the drafting progresses, the solvation completely disappears and the macromolecules move closer together. With the completion of this stage, the folded chain is transformed into a strandshaped chain structure formed by folding strands and straightening chains. The new entanglement structure Nodes quickly produce a large number of restrictions on the fiber elongation, while crystallinity, orientation, density, etc. also greatly increased and tends to be constant.

The crystal orientation of the fiber at high temperatures is a slow process. If the rate of stretching is too high, the fiber will not be drawn in sufficient stretching orientation, which is detrimental to the crystallization of the fiber. If the rate is too slow, the fiber will be burned at high temperatures The temperature will also affect the fiber drawing process, the temperature is too high will destroy the molecular structure of the drawing effect, the temperature is too low can not make the fiber chain fully stretched under stress, it is necessary to explore the appropriate Spinning rate and suitable spinning temperature.

\section{Conclusion}

In this paper, a modified UHMWPE raw material was prepared by using the process route of independent intellectual property. The UHMWPE fiber was prepared by the jelly spinning method. The process of the UHMWPE fiber and the change of the microstructure of the UHMWPE fiber in the preparation process were studied.

\section{References}

[1] Marissen R. Design with ultra strong polyethylene fibers[J]. Materials Sciences and Applications, 2011,2(5):319-320.

[2] Kavesh S, Prevorsek D C. Ultra high strength, high modulus polyethylene spectra fibers and composites[J]. International Journal of Polymeric Materials, 1995,30(2):15-56.

[3] Suñer S, Joffe R, Tipper J L, et al. Ultra high molecular weight polyethylene/graphene oxide nanocomposites: Thermal, mechanical and wettability characterization [J]. Composites Part B: Engineering, 2015,78(2):185-191.

[4] Prevorsek D C, Chin H B, Bhatnagar A. Damage tolerance: design for structural integrity and penetration[J]. Composite Structures, 1993,23(2):137-148.

[5] Wool R P, Bretzlaff R S, Li B Y, et al. Infrared and Raman spectroscopy of stressed polyethylene[J]. Journal of Polymer Science Part B: Polymer Physics, 1986,24(3):1039-1066. 